\title{
LES EFFORTS D'ORGANISATION ET D'ASSAINISSEIMENT DU MARCHÉ DU LAIT EN FRANCE
}

\author{
par A. TAPERNOUX \\ Professeur de Chimie à l'Erole Vétérinaire de Lyon. \\ A la mémoire \\ de mon Maître Ch. PORCHER.
}

Après de nombreux pays étrangers, qui profitent déjà des bierfaits d'une organisation méthodique de la production laitière, les Pouvoirs publies français, suivant les vœux des intéressés : producteurs, industriels et commerçants, consommateurs, cherchent, dans un effort très louable d'organisation et d'assainissement du marché, un remède à la crise qui sévit actuellement sur la production laitière, et qui menace de s'aggraver, si des mesures rationnelles ne sont pas mises en ouvre au plus tôt.

Pour ceux qui, comme moi, ont suivi depuis la naissance de la Revue Le Lait, l'œuvre admirable, dans son ensemble, du Maître qui l'a fondée, le très regretté Professeur Ch. Poncher, dont je m'honore d'avoir été l'élève fidèle, la question débattue en cette période peut n'apparaître pas nouvelle. Au cours d'un apostolat de tous les instants, jusqu'à la dernière minute de sa vie, et jusqu'à l'extrême limite de ses forces, mon Maître, Ch. Porcher, a lutté, par la plume, par la parole, par l'action, pour cette organisation et pour cet assainissement.

Cette grande voix s'est tue, nous laissant le glorieux et lourd héritage, à nous, ses élèves affectionnés, de poursuivre la tâche entreprise et de lutter, comme il avait lutté, pour la bonne cause, celle qui réunit en une noble synthèse un problème social des plus graves et une question d'économie nationale des plus importantes.

Il aurait, j'en suis sûr, applaudi aux efforts actuellement tentés, et apporté les suggestions intéressantes que lui dictaient sa passion généreuse et sa froide logique scientifique pour tout ce qui touche le problème du Lait.

On pourrait retrouver dans sa Revue, sous sa plume, la plupart des mesures actuellement envisagées et leur critique, ses appels et ses prédictions. Malheureusement, et suivant la formule célèbre, le moment n'est plus de regarder en arrière ; il faut, après avoir pesé tous les éléments du problème, appliquer ces mesures, que la situation actuelle de notre économie laitière rend indispensables et urgentes. Que ce juste hommage rendu au précurseur, soit la légitime récompense de sa clairvoyance, et qu'il soit, pour nous tous, eeux qui l'aimions et qu'il aimait, une source nouvelle d'ardeur. 


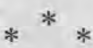

Une très vieille discipline, qui est, je crois, la meilleure, indique que la première des conditions, celle qui est indispensable pour résoudre un problème, c'est d'en poser convenablement les données. Quels sont donc les éléments du problème du marché du lait en France? Quelles sont les données qui permettront d'essayer d'apporter des solutions rationnelles? Telles sont les questions auxquelles j'essaierai de répondre avant toute déduction.

Pour plus de clarté, je résume en quelques propositions les éléments essentiels du débat :

$1^{\circ}$ Pour le producteur, le lait et les produits laitiers, - suivant en cela la plupart des productions de l'économie agricole, - ont subi au cours de ces dernières années une forte baisse; qui, malgré les mesures de protection qui ont été édictées, ne lui permet pas de retirer de l'exploitation des animaux laitiers la juste rémunération, non seulement de sa peine et de son travail, mais encore du capital engagé et des risques qu'il doit couvrir. Cette situation déficitaire menace de s'aggraver au cours de l'année qui commence.

$2^{\circ}$ Pour le consommateur, le lait et les produits laitiers représentent évidemment un appoint sérieux dans l'établissement de sa ration; mais, pour des raisons diverses, - les unes économiques, les autres hygiéniques et diététiques, - on peut dire qu'il ne tire pas suffisamment parti de ce produit national, et que, s'il le voulait, il pourrait facilement aider à conjurer la crise actuelle, crise dont il souffre lui-même par répercussion.

$3^{\circ} \mathrm{L}^{\prime}$ industriel et le commerȩant, eux-mêmes, étapes nécessaires entre la production et la consommation, sont touchés par la diminution de leur volume d'affaires et par la difficulté qu'ils éprouvent à écouler les produits de transformation.

Enfin, cette situation générale, que j’ai considérée comme un processus évoluant en vase clos, sur le territoire national, est dominée par des facteurs internationaux, la baisse des cours des produits laitiers étrangers, portant un coup redoutable à nos exportations, et nécessitant la mise en œuvre de mesures de protection dont la rigidité, nécessaire, a des répercussions fatales dans d'autres domaines.

\section{LA PRODUCTION FRANĢAISE}

Dans sa thèse de Doctorat vétérinaire, soutenue en 1928, et réalisée sous l'éminente direction de mon collègue et ami le Professeur E. LetaRd, le Docteur Vétérinaire Duchet évaluait à 7.400 .000 le nombre de vaches, dont $70 \%$ traites pour la consommation (soit approximativement 5.200.000). 
En 1934, J. Vienlly, docteur vétérinaire à Grenoble, donne, dans sa thèse, les chiffres suivants pour l'année 1932 : 261.000 taureaux ; 1.374 .000 bœufs ; 8.451 .000 vaches laitières ; 3.096 .000 élèves de plus d'un an ; 2.460 .000 élèves de moins d'un an ; soit, au total, 15.642.000 têtes de bétail bovin.

Le très regretté Professeur DechambRe, dans ce très bel ouvrage édité en 1934 par l'Office National de Propagande du Lait, beurre et fromage, et intitulé "La France Laitière ", donne les chiffres suivants : sur 15.829 .790 bovins, la France possède 8.572 .490 vaches, dont 5.500.000-6.000.000 sont traites pour l'alimentation humaine.

Voici d'autre part, et pour donner une idée rétrospective, une statistique de l'effectif du eheptel bovin franȩais depuis 1840, statistique communiquée par mon collègue et ami le Docteur Vétérinaire Jean BLAIN, chargé du cours de Zootechnie à l'Ecole Vétérinaire de Lyon :

$\begin{array}{llll}1840 \ldots \ldots \ldots \ldots \ldots & 11.761 .590 & 1926 \ldots \ldots \ldots \ldots \ldots & 14.482 .440 \\ 1897 \ldots \ldots \ldots \ldots \ldots & 13.486 .519 & 1927 \ldots \ldots \ldots \ldots \ldots & 14.940 .960 \\ 1909 \ldots \ldots \ldots \ldots \ldots & 14.297 .570 & 1929 \ldots \ldots \ldots \ldots \ldots & 15.630 .140 \\ 1913 \ldots \ldots \ldots \ldots \ldots & 14.787 .710 & 1930 \ldots \ldots \ldots \ldots & 15.467 .460 \\ 1918 \ldots \ldots \ldots \ldots \ldots & 12.250 .800 & 1931 \ldots \ldots \ldots \ldots \ldots & 15.433 .840 \\ 1924 \ldots \ldots \ldots \ldots \ldots & 14.024 .960 & 1934 \ldots \ldots \ldots \ldots \ldots & 15.704 .480\end{array}$

D'autre part encore, voici un tableau statistique concernant la variation du nombre des vaches de 1925 à 1930 (y compris 200.000 vaches pour l'Alsace-Lorraine) :

\begin{tabular}{|c|c|c|c|}
\hline & $\begin{array}{l}\text { Nombre } \\
\text { des vaches }\end{array}$ & & $\begin{array}{c}\text { Nombre } \\
\text { des vaches }\end{array}$ \\
\hline $1913 \ldots \ldots \ldots$ & 7.794 .270 & $1930 \ldots \ldots \ldots \ldots \ldots$ & 8.288 .000 \\
\hline $1925 \ldots \ldots \ldots \ldots$ & 7.500 .000 & $1931 \ldots \ldots \ldots \ldots$ & 8.274 .400 \\
\hline $1926 \ldots \ldots \ldots \ldots$ & 7.701 .000 & $1932 \ldots \ldots \ldots \ldots$ & 8.457 .220 \\
\hline $1927 \ldots \ldots \ldots \ldots \ldots$ & 7.971 .000 & $1933 \ldots \ldots \ldots \ldots \ldots$ & 8.572 .490 \\
\hline $1928 \ldots \ldots \ldots \ldots \ldots$ & 8.118 .000 & $1934 \ldots \ldots \ldots \ldots \ldots$ & 8.653 .190 \\
\hline $929 \ldots \ldots \ldots \ldots$ & 8.195 .000 & & \\
\hline
\end{tabular}

A ces chiffres, il faut ajouter 5.928 .540 brebis laitières et 1.400 .000 chèvres:

Quelle est maintenant la production en lait de ce cheptel national ? En éliminant immédiatement les petits ruminants, dont la production annuelle est la suivante :

Pour l'espèce ovine, 965.000 hectolitres, dont 730.000 transformés en fromages (DECHAMBRE) ;

Pour l'espèce eaprine, 2.600.000 hectolitres, dont 1.100 .000 transformés en fromages (DECHAMBRE),

on peut donner les évaluations suivantes pour la production bovine. 
D'après M. le Ministre H. QubuILLE ("La France Laitière »), les destinations du lait ont été, en ces dix dernières années, réparties à peu près eomme il suit :

29.400.000 hectolitres ont servi à l'alimentation des veaux (soit $21 \%$ ).

42.000.000 d'hectolitres ont servi à la vente du lait en nature (soit $31 \%$ ).

49.000.000 d'hectolitres ont servi à la fabrication du beurre (soit $34 \%$ ).

19.600.000 hectolitres ont servi à la fabrication des fromages (soit 14\%).

Soit, au total, une production globale moyenne de 140 millions d'hectolitres.

Si l'on admet le chiffre de 140 millions d'hectolitres et que l'on adopte le nombre de 6 millions de vaches traites pour l'alimentation humaine (chiffre donné par DEсHAMBRE), on arrive à un rendement annuel de 2.300 litres environ, rendement qui me semble un peu fort; en réalité, et en tenant compte que le chiffre de Dechambre est un peu faible, on peut admettre que le rendement annuel moyen par vache ne dépasse pas 2.000-2.100 litres.

Ce qu'il y a de particulièrement intéressant à considérer, c'est la répartition de l'effectif des vaches, et par conséquent, de la production ; d'après M. le Ministre QueUILLE («La France Laitière»), on trouve $10 \%$ seulement de l'effectif dans les fermes possédant plus de 20 vaches, $12 \%$ dans les fermes possédant de 5 à 20 vaches et $78 \%$ dans des fermes possédant moins de 5 têtes; les soins de ce bétail occupent ehaque jour plus de 1.530 .000 foyers ruraux, et d'après M. le Sénateur Donon, 4 millions de familles équilibrent leur budget avec la production laitière et tirent de cette production leur principal moyen d'existence dans 50 départements, sur 89.

Le revenu brut de cette production est évalué d'après M. le Sénateur Donon, à près de 10 milliards de franes (sommes encaissées par les producteurs); cette évaluation, qui fait ressortir le prix du litre, en moyenne, à 0,70 , n'est plus atteinte actuellement.

On a dit, à juste titre, et répété, que le paiement du lait apportait au foyer rural les revenus journaliers, hebdomadaires ou mensuels qui représentent les salaires pour d'autres classes sociales ; alors que les productions végétales et animales autres que le lait, sont des productions à longue échéance dont le revenu est annuellement perçu, la vente du lait et des produits laitiers permet à l'agriculteur d'attendre ces échéances et d'assurer son pouvoir d'achat pour les divers aliments ou objets manufacturés qui lui sont nécessaires ; on voit aisément les répercussions désastreuses qui découlent 
d'une baisse massive ou d'une mévente portant sur le lait et les produits laitiers; il devient alors un chômeur partiel ou total, il n'achète plus et accentue la crise qui le frappe en la généralisant ; il sent germer en lui ces ferments de révolte, et sans regarder plus avant, il établit le parallèle entre les prix de vente de son lait et les prix de vente à la consommation; il ne voit pas que si même le lait avait une valeur nulle à la production, le consommateur urbain serait obligé de l'acheter à un prix déterminé pour couvrir les frais de ramassage, de transport, de transformation, les impôts et les frais de vente. C'est pourquoi il faut à tout prix apporter remède à une situation qui risque de s'établir; toute amélioration dans ce domaine constitue un remède à la crise générale de l'économie nationale; toute aggravation, au contraire, signifierait une recrudescence inquiétante de cette même crise.

Mais poursuivons cette étude des données du problème avant de nous engager plus avant dans le débat.

II résulte incontestablement des chiffres cités, que le nombre des animaux de l'espèce bovine a augmenté progressivement au cours de ces dernières années. La dernière statistique publiée au Journal officiel (du 29 mars 1935 ; page 3572 ) accuse 15.704.480 têtes de bovins en 1934, et marque un léger recul sur l'année précédente ; toutefois, cet effectif global reste supérieur de près d'un million de têtes à celui de 1913. Cette augmentation, dont il faut d'ailleurs déduire la population bovine d'Alsace-Lorraine, entraine forcément et parallèlement une augmentation du nombre d'hectolitres de lait produits ; mais il faudrait bien se garder de croire que la quantité de lait produite en plus, est proportionnelle à l'augmentation de l'effectif bovin; en réalité, - et c'est là un fait d'ordre général, - la production d'après guerre s'est orientée vers les races à grand rendement quantitatif, mais à faible rendement qualitatif, comme pour le blé, le vin et d'autres produits; le cheptel détruit pendant la guerre a été reconstitué sur ces bases, et la mesure n'a pas été suffisamment gardée ; il en résulte que l'augmentation de la quantité de lait est proportionnellement plus grande que l'augmentation de l'effectif bétail. J'exposerai d'ailleurs, dans ce travail, les conséquences de cette orientation de la production. Qu'il suffise pour l'instant de comprendre que, le lait étant payé très généralement au litre ou au kilog, c'est-à-dire à la quantité et non à la qualité, ce qui peut paraître paradoxal, la tendance naturelle du producteur est de suivre l'exemple de celui qui, ayant substitué dans son troupeau des vaches à grand rendement aux vaches autochtones, produit avec les mêmes frais une quantité de lait plus grande par animal et par jour; la conséquence inévitable de cette tendance, e'est la course à la surproduction, c'est-à-dire à la baisse des cours. Finalement, avee plus de peine et en manipulant une plus grande quantité de 
produit, le producteur ne reçoit pas plus d'argent; c'est ce qu'on peut dénommer le mirage des races à grand rendement.

\section{2。 LA CONSOMMATION DU LAIT EN FRANCE}

Il faut distinguer la consommation du lait en nature et la consommation du lait sous forme de produits dérivés.

\section{a) Lait en nature.}

Il résulte des chiffres précédemment fournis, que 42 millions d'hectolitres vont en France directement au consommateur sans avoir été transformés; cela représente approximativement 0 1. 3 par personne et par jour, à peine un tiers de litre!

Dans un document, fort intéressant, publié en 1926 ( Le ravitaillement en lait d'une grande ville française »), P. SAINT-OLIVE et R. Guyot-Sionnest donnent pour l'année 1923 et pour quelques villes, les chiffres qui suivent :

\begin{tabular}{|c|c|c|c|}
\hline & $\begin{array}{l}\text { Par habitant } \\
\text { et par jour }\end{array}$ & & $\begin{array}{c}\text { Par habitant } \\
\text { et par jour }\end{array}$ \\
\hline Annecy .. & 01.27 & Mulhouse & 01.60 \\
\hline Bordeaux & 01.16 & Nantes. & 01.20 \\
\hline Chambéry & 01.26 & Nice ... & 01.26 \\
\hline Clermont-Ferrand ... & 01.15 & Paris ............. & 01.33 \\
\hline Grenoble ........ & 01.26 & Roanne .......... & 01.40 \\
\hline Lille ......... & 01.22 & Rouen & 01.12 \\
\hline Lyon & 01.17 & Strasbourg ........ & 01.41 \\
\hline 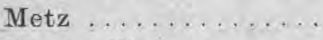 & 01.40 & Troyes ........... & 01.25 \\
\hline Montpellier ........ & 01.10 & Vienne............. & 01.24 \\
\hline
\end{tabular}

soit, en moyenne, 0 1. 26 (par habitant et par jour). On peut donc dire, sans grosse chance d'erreur, que la consommation française de lait en nature au cours de ces dix dernières années, est d'un peu plus un quart de litre et d'un peu moins un tiers de litre par habitant et par jour.

La Revue Le Lait (1933; page 528) indiquait, d'après la Revue de Zootechnie, une consommation de 114 litres de lait en nature par tête d'habitant et par an, ce qui donne la moyenne journalière de 0 1. 31.

On peut utilement rapprocher ces chiffes de ceux qui sont publiés dans les pays étrangers ; dans les documents précédemment cités, nous trouvons les renseignements suivants :

\begin{tabular}{|c|c|c|}
\hline Pays & $\begin{array}{l}\text { Consommation } \\
\text { annuelle moyenne }\end{array}$ & $\begin{array}{c}\text { Consommation } \\
\text { par jour }\end{array}$ \\
\hline Allemagne & 124 & 01.33 \\
\hline Etats-Unis & 251 & 01.68 \\
\hline Finlande............. & 381 & 11. 04 \\
\hline Norvège $\ldots \ldots \ldots \ldots \ldots$ & 254 & 01.69 \\
\hline
\end{tabular}


Dans les grandes villes étrangères, en 1930, la consommation par tête et par jour est la suivante :

\begin{tabular}{|c|c|c|c|}
\hline New-York. . . . . . . . . . & 01.85 & Munich & 01. 39 \\
\hline Zurich $\ldots \ldots \ldots \ldots \ldots$ & 01.62 & Budapest ...... & 01.36 \\
\hline Vienne............... & 01.48 & Dresde............... & 01.27 \\
\hline Copenhague. & 01,45 & Berlin & 01.26 \\
\hline
\end{tabular}

La consommation à Berlin serait tombée à 01.22 en 1932 et aurait encore diminué en 1933 (Le Lait, 1933 ; page 1070).

On doit reconnaître, par conséquent, que la consommation de lait en nature est particulièrement faible dans notre pays si on la compare à celle des pays gros producteurs de lait. D'où provient cette sous-consommation?

On ne peut valablement soutenir pour le lait en nature, que la sous-consommation provient de la crise actuelle; elle existait avant la crise, et celle-ci n'a pu qu'accentuer un état de fait déjà établi.

On peut invoquer plusieurs causes qui concourent à ce délaissement progressif : d'abord, le peu de soin apporté à la récolte, au transport, et à la livraison du lait, a peu à peu éloigné le consommateur d'une marchandise parfois de mauvaise qualité ; la facilité de la fraude, la publicité intempestive donnée aux actes de répression de cette fraude, publicité qui n'a, je erois, aucunement l'effet de l'exemple, ont pu amener le consommateur à penser que le liquide qu'il achetait sous le nom de lait, était mauvais parce que malpropre ou falsifié ou encore malpropre et falsifié tout à la fois.

Pour accentuer les conséquences de cet état d'esprit, certains médecins se sont mis de la partie. Après avoir montré, avec juste raison, les dangers que peuvent faire courir au consommateur certains laits porteurs de germes ou de produits nocifs qui proviennent soit de l'animal laitier, soit de pollutions postérieures à la traite, ils ont déconseillé et parfois même interdit la consommation du lait frais en nature; il semble, d'ailleurs, que la période de cette interdiction étant révolue, on se rende compte de l'excès dans lequel on était tombé.

Une preuve de cet état d'esprit regrettable, nous est donnée par le texte même du projet de loi tendant à l'organisation et à l'assainis sement du marché du lait adopté par la Chambre des Députés. L'article 6 prévoit un traitement d'assainissement pour les laits de consommation courante (pasteurisation, stérilisation, etc.). Qu'on assainisse le marché du lait, je veux bien, mais qu'on dise au consommateur, le lait que vous consommez a subi un traitement d'assainissement dans un atelier d'assainissement, il ne faut pas être très grand psychologue pour prévoir la réaction de ce consomma- 
teur; il s'abstiendra de consommer, jugeant que la marchandise qu'on lui offre est mauvaise, puisqu'elle a besoin d'être assainie.

C'est en pratiquant l'usage des expressions péjoratives, dont la précédente est un petit exemple, qu'on se fait une popularité éphémère, mais c'est ainsi que l'on porte un coup mortel à la cause que l'on prétend défendre.

L'emploi des expressions péjoratives est une calomnie : ealomnie pour le producteur, qui se débat dans les affres d'une existence parfois dure, et qui, mon Dieu! ne fait peut être pas toujours au mieux parce $q u$ 'il est peu conseillé et irrationnelllement soutenu; calomnie pour ceux qui, auxiliaires du producteur, s'efforcent d'améliorer la qualité du lait et des produits laitiers; calomnie enfin, pour ce produit national quí devrait être un aliment national, comme il l'est en Suisse, et qui, par ses qualités nutritives et son bas prix relatif, est spécifiquement un aliment de crise.

Il est bien certain, que le lait tel qu'il est produit actuellement, n'est pas parfait; il est nécessaire de l'améliorer, et je montrerai comment je conçois cette amélioration ; mais je tiens à insister encore sur une des causes de la désaffection présente du consommateur pour le lait. J'ai parlé plus haut des vaches à grand rendement quantitatif, et j'ai essayé de montrer, au point de vue énonomique, combien le résultat de leur exploitation était mauvais. Mais, du point de vue consommation en nature, le résultat de cette exploitation est bien plus mauvais encore; je fais appel à tous ceux qui ont dégusté, et qui au laboratoire ont analysé de ces laits; ces laits ne présentent certes pas la saveur des laits riches en beurre et en extrait dégraissé, et présentent une valeur nutritive moindre; je sais bien que certains médecins prétendent que la forte teneur du lait en beurre peut fatiguer le foie, et qu'il vaut mieux un lait faible en matière grasse, lait demi-écrémé par exemple. Voire ! Il ne faut peut-être pas partir du cas particulier du malade pour généraliser aux bien portants. Et il est bien facile de comprendre que le consommateur, devant la platitude de ce que VimıLy appelle certains crus de lait, crus qui tendent à se généraliser, réduise cette consommation au strict minimum, lorsqu'il ne la supprime pas complètement. Et alors, irrémédiablement ce consommateur est perdu pour la cause du lait, les efforts de propagande se heurtent chez lui à la force invincible de l'habitude.

\section{b) Produits dérivés du lait.}

D'après la statistique donnée par Le Lait (1933; page 528), que j'ai citée plus haut, le Français consommerait en moyenne par an, $3 \mathrm{~kg} .86$ de beurre et $4 \mathrm{~kg}$. 77 de fromages.

En admettant qu'il faille en moyenne 22 litres de lait pour obtenir 
1 kilogramme de beurre, et 8 litres de lait pour obtenir 1 kilogramme de fromage, nous avons le tableau suivant pour les différents pays :

\begin{tabular}{|c|c|c|c|c|}
\hline Pays & $\begin{array}{l}\text { Consommation } \\
\text { en nature }\end{array}$ & $\begin{array}{l}\text { Kilogrammes } \\
\text { de beurre }\end{array}$ & $\begin{array}{l}\text { Kilogrammes } \\
\text { de fromages }\end{array}$ & $\begin{array}{c}\text { Consommation } \\
\text { totale (en litres } \\
\text { de lait) }\end{array}$ \\
\hline Allemagne ........ & 124 & 8,93 & 5,81 & 365 \\
\hline Etats-Unis ........ & 251 & 7,85 & 1,86 & 440 \\
\hline Finlande........ & 381 & 9,50 & $1,4.5$ & 600 \\
\hline France ........ & 114 & 3,86 & 4,77 & 235 \\
\hline Norvège & 254 & 4,36 & 4,85 & 390 \\
\hline
\end{tabular}

En adoptant ces chiffres comme base de calcul, on trouve approximativement que le Français utilise 34 millions d'hectolitres de lait sous forme de beürre, sur les 49 millions utilisés à cette fabrication, et qu'il consomme 15.280 .000 hectolitres sous forme de fromages, sur les 19.600 .000 utilisés à cette production.

En outre, il faut tenir compte des importations et des exportations, dont voici les éléments pour les premiers semestres de 1933 et de 1934 (Bulletin de l'Institut International du Froid, 1934, n ${ }^{\circ} 6$, p. 707 et 708$)$ :

Beurre (tonnes métriques).

$1^{\mathrm{er}}$ semestre de 1934

1. 521

3.981 $1^{\text {er }}$ semestre de 1933

1. 505

7. 237

$\begin{array}{lrl}\text { Exportation . . . . . . . . } & 1.521 & 1.505 \\ \text { Importation . . } & 3.981 & 7.237\end{array}$

Fromages (tonnes métriques).

$1^{\text {er }}$ semestre de 1934

$\begin{array}{lrr}\text { Exportation . . . . . . } & 6.044 & 6.038 \\ \text { Importation . . . . . } & 7.167 & 12.490\end{array}$

$\begin{array}{lrr}\text { Exportation } \ldots \ldots \ldots & 6.044 & 6.038 \\ \text { Importation . . . . . } & 7.167 & 12.490\end{array}$

ler semestre de 1933

6.038

$\begin{array}{lrr}\text { Exportation . . . . } & 6.044 & 6.038 \\ \text { Importation . . . . } & 7.167 & 12.490\end{array}$

Ce tableau est éloquent, car il montre que, malgré la surproduction, nous avons importé pendant une partie des années 1933 et 1934, plus que nous n'avons exporté - de marchandises dont nous avions déjà trop.

\section{$3^{\circ}$ LES PRINCIPES D'UNE ORGANISATION RATIONNELLE DU MARCHÉ DU LAIT}

Après avoir objectivement envisagé les données du problème, je vais essayer de construire un système logique de mesures destinées à favoriser la lutte contre la crise et à enrayer celle-ci.

$\mathrm{Au}$ préalable, il doit être bien entendu, que rien ne sera réalisé sans un effort de discipline collective, sans que tout le monde soit pénétré de cette idée que sa collaboration à l'œuvre entreprise est utile et qu'il doit la donner entièrement.

Les mesures que l'on peut appliquer visent la production, l'industrie et le commerce et la consommation. 


\section{a) Production.}

Les mesures qui concernent la production, doivent avoir pour effet d'agir favorablement sur la quantité et sur la qualité du lait produit.

Pour résorber l'excédent de production, un certain nombre de mesures ont été envisagées ; les plus efficaces sont celles qui tendent à orienter la production vers une plus forte teneur en principes utiles, et particulièrement à fixer un minimum régional pour la teneur en beurre des laits vendus au commerce et au consommateur. Cette mesure, dans laquelle certains cherchent à voir une source de tracasseries pour le producteur, est en réalité conforme à son intérêt bien compris, à celui de l'industriel et à celui du consommateur.

Pour le producteur, et indépendamment de l'indication très nette de l'orientation nouvelle qui lui est demandée, elle supprimerait la plupart de ces stupides procès pour écrémage, auxquels on pourrait, sans nul inconvénient, substituer le système, plus souple, des avertissements, des amendes et du refus pur et simple de la livraison.

L'industriel et le commerçant verraient progressivement se résorber les trop-pleins déficitaires; travaillant une marchandise meilleure, leurs prix de revient seraient abaissés, d'où baisse progressive et sensible des prix de vente des produits fabriqués au consommateur. Celui-ci, enfin, en dehors du bénéfice réalisé par la baisse précédente, serait attiré de plus en plus à la consommation en nature d'un lait plus savoureux. En résumé, on peut prévoir, de l'application progressive de cette seule mesure, une diminution finale de la quantité de lait mise sur le marché, doublée d'une augmentation parallèle de la consommation du lait en nature et des produits dérivés, les deux effets agissant dans le même sens pour résorber l'excédent et pour maintenir les cours ou même les élever à la production. Il est bien évident que l'amélioration serait progressive et qu'il ne faut pas espérer rétablir une situation chaotique par un coup de baguette magique.

Mais il faudra bien en arriver à prendre des mesures, si l'om ne veut que le chaos continue; et plus tardivement elles seront prises, plus tardivement les effets bienfaisants se feront sentir. Rien n'empêcherait du reste, pour ménager tous les intérêts des producteurs, de graduer progressivement ce minimum, qui pourrait être relevé périodiquement, de façon à leur laisser le temps matériel d'apporter à leur élevage les modifications qui s'imposent.

Les autres mesures destinées à lutter contre l'excédent, sont également prévues; elles comprennent notamment la suppression de la vente des laits écrémés ou demi-écrémés pour la consommation humaine en nature, la fixation d'un taux élevé de matière grasse 
pour les produits laitiers, - et notamment pour les fromages, une active propagande en faveur de la consommation plus large du lait en nature, propagande qui doit s'exercer, non seulement par les méthodes modernes de la publicité, mais encore par l'exemple dans les établissements d'éducation, dans les hôpitaux, les casernes, etc.

Mais pour que le rendement de l'effort qui vient d'être décrit soit maximum, un autre point, des plus importants, doit être souligné : il s'agit de la qualité du lait au point de vue de l'hygiène. Dans ce domaine encore, rien n'est possible sans la collaboration effective du producteur, de l'industriel et du commerçant, dont les intérêts convergents sont encore ceux du consommateur. Au producteur, il faut demander d'écouter avee attention et d'appliquer consciencieusement les principes qui lui seront inculqués pour améliorer ses méthodes de travail; certains, peu nombreux, se sont volontairement soumis à un contrôle officiel, que leur a proposé le ministre intéressé dès 1927, ce qui montre, en passant, que les Pouvoirs publics ont depuis longtemps compris les nécessités, qui deviennent plus urgentes maintenant. Ces producteurs bénévoles doivent être félicités hautement, car leur geste n'a peut-être pas encore reçu la sanction économique qu'il méritait. On trouvera dans cette Revue (1934; page 902), sous la signature d'un de ces pionniers de l'amélioration de la production laitière, Jean PorCHerel, la relation de einq années de contrôle facultatif appliqué dans une région du Centre. Il en est d'autres, plus grands ou plus petits, suivant la quantité de lait traitée, mais qui sont tous aussi grands par l'exemple qu'ils nous donnent. Il faut done encourager ces initiatives, les développer par tous les moyens, et essayer, malgré les diffieultés inhérentes à la petite production, de les généraliser. Ceux-là peuvent et doivent vendre leur lait cru. Mais ce travail de généralisation est un travail de longue haleine, comme tout ce qui touche à l'éducation persuasive. Il faut en attendant, parer au plus pressé, c'est-à-dire, tout en essayant d'améliorer progressivement la production actuelle en élevant son standard hygiénique, éviter la possibilité d'accidents qui, s'ils se montrent peu fréquents, heureusement, peuvent laisser planer sur le lait une suspicion.

Dans l'état actuel de notre production nationale, je ne vois qu'une méthode, capable de faire l'intérim, jusqu'à l'amélioration souhaitée ; cette méthode, - si nous laissons de côté la stérilisation proprement dite, qui ne peut être généralisée, - e'est la pasteurisation. Il ne m'appartient pas, dans cet exposé, de vanter les effets de la pasteurisation moderne : on trouverait suffisamment d'arguments dans le cadre même de cette Revue, et notamment dans le numéro de janvier-février 1933. Il y a pour et contre cette méthode de traitement du lait, des partisans et des adversaires aussi profondément 
respectables dans leurs convictions, mais pour celui qui suit l'évolution des idées sur ce sujet depuis de nombreuses années, il est curieux de constater qu'à mesure que la technique s'est améliorée, d'excellents esprits se sont ralliés à la généralisation de son application, qui, pour moi, ne constitue qu'une étape, mais une étape indispensable.

L'éventualité d'approvisionnement généralisé par lait cru, ne serait souhaitable que s'il était possible d'obtenir partout un lait cru officiellement contrôlé.

Il faut done chercher à développer le plus possible la pasteurisation, tout en contrôlant les procédés mis en œuvre et leur efficacité ; e'est la voie dans laquelle on tend à s'engager, mais, malheureusement, il faut, là encore, reconnaître qu'une telle mesure n'est pas applicable partout, et notamment dans les campagnes, où les fruitières, les producteurs, vendent directement leur lait au consommateur. Il faut done également prévoir pour ces cas particuliers, des mesures qui permettront provisoirement d'apporter au consommateur rural une plus grande sécurité ; celle qui consisterait à conseiller au consommateur, au moment de la vente, de soumettre le lait qu'il achète à l'ébullition, me paraît fort raisonnable. En résumé, si l'on adopte les mesures précédemment étudiées, il ne resterait plus sur le marché - les laits stérilisés étant mis à part - que les types cidessous :

a) Les laits officiellement contrôlés crus ou pasteurisés ;

b) Les laits erus non contrôlés à soumettre à l'ébullition;

c) Les laits pasteurisés obtenus à partir des laits crus non contrôlés.

La pasteurisation pourrait même être étenđue à la fabrication des produits dérivés du lait : pour les beurres, la chose paraît réalisable, puisque, de plus en plus, l'industrie s'oriente vers la pasteurisation des crèmes destinées à cette fabrication; pour les crèmes, il n'y a aucune difficulté pratique; pour les fromages, au contraire, la question mérite d'être étudiée de près, et peut-être faut-il se borner, comme l'indiquait Fouassier (Revue d'Hygiène et de Médecine préventive, avril 1935 , p. 274 et suivantes), à préconiser la pasteurisation des laits destinés à la préparation des fromages frais, pour lesquels les fermentations ultérieures ou la cuisson de la pâte n'ont pas exercé leur action stérilisante.

Les résultats que l'on peut espérer de l'application des mesures précédentes, et notamment de la généralisation de la pasteurisation aux laits mélangés, sont actuellement prévisibles; il n'est besoin que de se reporter à l'exemple fourni par la Ville de Strasbourg.

Des comptes rendus statistiques de cette ville (de 1919 à 1933), 
nous avons extrait trois tableaux, qui montrent l'influence bienfaisante de la pasteurisation généralisée - mesure adoptée depuis 1915 et continuée après la guerre.

Le premier tableau indique les décès au cours de la première année pendant la période 1900-1933. Sur 100 enfants nés vivants, on voit que le pourcentage de mortalité, de 24,5 en 1900 , était encore de 11 en 1921 ; il a pu être amené à 4,81 en 1933.

Le deuxième tableau est réservé aux décès par diarrhée et entérite des enfants au-dessous de 2 ans pendant la période 1920-1933; on note également une baisse considérable, qui passe de 68,24 pour 100.000 habitants en 1920 à 8,65 en 1933. Enfin, le troisième tableau établit la comparaison de la mortalité infantile suivant les mois pendant deux périodes de 5 ans : 1900-1904 et 1928-1932. Ce tableau est particulièrement suggestif, en ce sens qu'au cours de la première période, les décès pendant les mois de chaleur (juillet et août) se montrent beaucoup plus nombreux que pendant les autres mois; ils sont près du double et quelquefois plus du double des décès pendant les mois froids (janvier et février). Au contraire, dans la deuxième période (1928-1932), alors que la température moyenne est comparable, les déeès en juillet et en août ne dépassént pas ceux des autres mois, et sont parfois inférieurs en nombre. Il n'est pas possible de ne pas établir un parallèle entre cette amélioration d'une situation qui apparaît très favorable actuellement, et la qualité du lait destiné à l'enfance. Strasbourg est un exemple à donner pour montrer les bons effets d'une réglementation bien conçue.

\section{Premier tableau.}

Sont décédés au cours de la première année à Strasbourg (Comptes rendus statistiques de la Ville de Strasbourg, p. 147, 148 et 168):

\begin{tabular}{|c|c|c|c|c|}
\hline & Année & Total & $\begin{array}{l}\text { Pourcentage } \\
\text { sur la totalité } \\
\text { des décès }\end{array}$ & $\begin{array}{l}\text { Pourcentage } \\
\text { sur } 100 \text { enfants } \\
\text { nés vivants }\end{array}$ \\
\hline 1900 & $\ldots \ldots \ldots$ & I. 090 & 34 & 24,5 \\
\hline 1901 & $\ldots \ldots \ldots$ & 954 & 30,8 & 20,2 \\
\hline 1902 & $\ldots \ldots \ldots$ & 1.003 & 33,2 & 21,4 \\
\hline 1903 & $\ldots \ldots \ldots$ & 1.053 & 32,7 & 23,1 \\
\hline 1904 & $\ldots \ldots \ldots$ & 966 & 29,7 & 20,3 \\
\hline 1905 & $\ldots \ldots \ldots$ & 1.001 & 30,1 & 21,3 \\
\hline 1906 & $\ldots \ldots \ldots$ & 909 & 28,6 & 19,1 \\
\hline 1907 & $\ldots \ldots \ldots$ & 796 & 25,5 & 17,3 \\
\hline 1908 & $\ldots \ldots$ & 786 & 25,7 & 16,8 \\
\hline 1909 & $\ldots \ldots \ldots$ & 765 & 24,9 & 17,8 \\
\hline 1910 & $\ldots \ldots \ldots$ & 657 & 23 & 15,6 \\
\hline 1911 & $\ldots \ldots \ldots$ & 686 & 23 & 16,8 \\
\hline 1912 & $\ldots \ldots \ldots$ & 520 & 19,1 & 13,1 \\
\hline
\end{tabular}




$\begin{array}{cccc}\text { Année } & \text { Total } & \begin{array}{c}\text { Pourcentage } \\ \text { sur la totalité } \\ \text { des décès }\end{array} & \begin{array}{c}\text { Pourcentage } \\ \text { sur } 100 \text { enfants } \\ \text { nés vivants }\end{array} \\ 1913 \ldots \ldots \ldots \ldots & 475 & 16,8 & 12,1 \\ 1914 \ldots \ldots \ldots & 496 & 16 & 13,1 \\ 1915 \ldots \ldots \ldots & 381 & 9,7 & 12,6 \\ 1916 \ldots \ldots \ldots & 233 & 7,5 & 10,3 \\ 1917 \ldots \ldots \ldots & 216 & 6,6 & 10,3 \\ 1918 \ldots \ldots \ldots & 266 & 6,4 & 12,7 \\ 1919 \ldots \ldots \ldots & 276 & 9,8 & 9,4 \\ 1920 \ldots \ldots \ldots & 480 & 17,5 & 11,1 \\ 1921 \ldots \ldots \ldots & 473 & 17,1 & 11 \\ 1922 \ldots \ldots \ldots & 348 & 13,4 & 8,9 \\ 1923 \ldots \ldots \ldots & 356 & 13,69 & 8,68 \\ 1924 \ldots \ldots \ldots \ldots & 323 & 11,63 & 7,36 \\ 1925 \ldots \ldots \ldots \ldots & 387 & 13,25 & 8,38 \\ 1926 \ldots \ldots \ldots \ldots & 348 & 12,26 & 7,67 \\ 1927 \ldots \ldots \ldots \ldots & 282 & 10,54 & 6,62 \\ 1928 \ldots \ldots \ldots \ldots & 318 & 11,34 & 7,08 \\ 1929 \ldots \ldots \ldots & 357 & 11,63 & 7,77 \\ 1930 \ldots \ldots \ldots & 292 & 10,16 & 6,24 \\ 1931 \ldots \ldots \ldots \ldots & 289 & 9,23 & 6,13 \\ 1932 \ldots \ldots \ldots \ldots & 281 & 9,56 & 5,73 \\ 1933 \ldots \ldots \ldots & 218 & 7,35 & 4,81\end{array}$

Deuxième tableau.

DÉCÈs PAR DIARRHÉ ET ENTÉRITE DES ENFANTS AU-DESSOUS DE 2 ANS DE IA VILLE DE STRASBouRg. (Comptes rendus statistiques de la Ville de Strasbourg, 1919-1933; p. 151 et 155.)

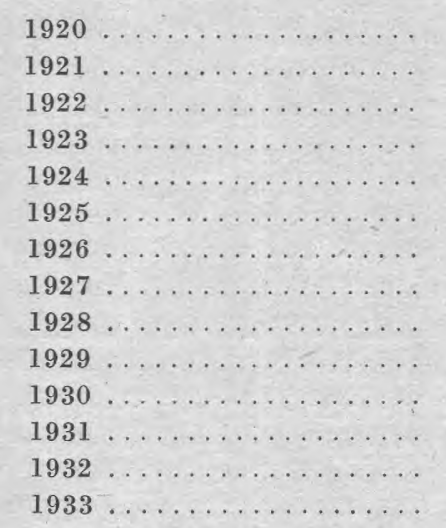

Par 100.000 habitants

$\begin{array}{rr}116 & 68,24 \\ 121 & 72,33 \\ 30 & 17,77 \\ 68 & 39,91 \\ 35 & 20,36 \\ 63 & 36,32 \\ 47 & 26,86 \\ 40 & 22,66 \\ 36 & 20,21 \\ 56 & 31,17 \\ 23 & 12,69 \\ 38 & 20,80 \\ 25 & 13,63 \\ 16 & 8,65\end{array}$




\section{Troisième tableau.}

TABLEAU COMPARATIF DE LA MORTALITÉ INFANTILE PENDANT LES PÉRIODES 1900-1904 ET 1928-1932, SUIVANT LES MOIS. (Page 175 des Comptes rendus statistiques de la Ville de Strasbourg.)

\begin{tabular}{|c|c|c|c|c|c|}
\hline . & 1928 & 1929 & 1930 & 1931 & 1932 \\
\hline Janvier & 65 & 70 & 47 & 72 & 58 \\
\hline Février $\ldots \ldots \ldots \ldots$ & 59 & 66 & 77 & 71 & 54 \\
\hline Mars ........... & 71 & 74 & 94 & 71 & 61 \\
\hline Avril $\ldots \ldots \ldots \ldots$ & 53 & 69 & 74 & 68 & 59 \\
\hline Mai .............. & 74 & 66 & 63 & 79 & 72 \\
\hline Juin ............ & 91 & 87 & 44 & 64 & 79 \\
\hline Juillet . . . . . . . & 187 & 154 & 88 & 110 & 178 \\
\hline Août $\ldots \ldots \ldots \ldots$ & 163 & 123 & 163 & 164 & 143 \\
\hline Septembre ........ & 95 & 71 & 123 & 126 & 78 \\
\hline Octobre .......... & 84 & 64 & 74 & 114 & 57 \\
\hline Novembre ........ & 82 & 52 & 77 & 53 & 65 \\
\hline Décembre......... & 66 & 58 & 79 & 64 & 62 \\
\hline tan & 1900 & 1901 & 1902 & 1903 & 1904 \\
\hline Janvier .......... & 23 & 28 & 22 & 40 & 21 \\
\hline Février $\ldots \ldots \ldots \ldots$ & 33 & 48 & 26 & 36 & 35 \\
\hline Mars ............. & 52 & 40 & 33 & 38 & 38 \\
\hline Avril $\ldots \ldots \ldots \ldots$ & 37 & 30 & 43 & 36 & 39 \\
\hline Mai ............ & 25 & 27 & 19 & 24 & 31 \\
\hline Juin ........... & 31 & 26 & 26 & 25 & 21 \\
\hline Juillet .......... & 25 & 24 & 27 & 13 & 10 \\
\hline Août $\ldots . . . \ldots \ldots$ & 26 & 32 & 16 & 21 & 24 \\
\hline Septembre ........ & 13 & 22 & 17 & 17 & 12 \\
\hline Oetobre .......... & 15 & 27 & 15 & 12 & 16 \\
\hline Novembre ........ & 22 & 24 & 14 & 15 & 19 \\
\hline Décembre......... & 18 & 29 & 34 & 11 & 15 \\
\hline
\end{tabular}

\section{b) Industrie et Commerce.}

Les mesures qui visent l'industrie et le commerce du lait peuvent être classées ainsi qu'il suit :

$1^{0}$ Amélioration des transports du lait, soit du centre de production aux stations de traitement, soit des stations de traitement aux centres de consommation.

$2^{\circ}$ Réalisation d'une pasteurisation efficace dans les meilleures conditions techniques et hygiéniques.

$3^{\circ}$ Application de soins méticuleux depuis la pasteurisation jusqu'à la livraison au consommateur.

Sur le premier point, la technique moderne a déjà apporté un certain nombre de réalisations intéressantes : réfrigération aussitôt après la traite, transport du lait en wagons ou en camions frigorifiques ou isothermes (qui permettent de le maintenir à basse tempé- 
rature), utilisation des citernes, création de gares laitières pour les manipulations délicates, etc. Il faut développer et encourager les efforts dans cette voie.

En ce qui concerne la pasteurisation, il est tout à fait souhaitable de la voir effectuer avec un contrôle, dont les modalités pourront être fixées par les Pouvoirs publics. Mais les résultats favorables obtenus par ce traitement, devront être suivis de soins méticuleux jusqu'à la livraison du lait au consommateur. Le meilleur système consiste dans la mise en bouteilles immédiate après la pasteurisation ; cette mesure doit être développée le plus possible,mais, comme son application entraînerait vraisemblablement une augmentation de prix, il serait peut-être opportun d'attendre des circonstances plus favorables pour la généraliser, tout en favorisant dès maintenant les expériences tentées ou à tenter.

Dans le cas où cette mesure ne peut être appliquée, la livraison en bidons restera la règle, depuis l'usine jusqu'au détaillant, et c'est dans cette livraison et cette distribution qu'il faudra ohercher à améliorer, par la voie d'un contrôle édueatif, et notamment éviter les pollutions multiples du lait, dues à la promiseuité de marchandises poussiéreuses, à l'action du vent, aux négligences des commerçants et des acheteurs, et, en été, aux mouches; etc.

\section{c) Consommation.}

Enfin, le lait arrivé à l'étape de la vente au consommateur, un dernier filtre peut être envisagé : c'est le contrôle hygiénique à la consommation. Déjà mis en œuvre dans certaines villes, il permettra d'avoir une idée des défectuosités existantes, de remonter à leur source et d'améliorer le produit vendu. En développant, suivant un rythme analogue, la propagande pour la consommation plus grande du lait, en multipliant les essais déjà tentés de distribution de lait aux enfants des écoles, en donnant à la campagne entreprise le sens social et national qu'elle doit avoir, nul doute que le consommateur, mieux protégé, mieux défendu, s'apercevra que son intérêt direct et indirect lui commande d'aider les efforts qui sont faits pour lui assurer la livraison d'un aliment de tout premier ordre par ses qualités nutritives.

\section{GONGLUSIONS}

Les efforts d'organisation et d'assainissement du marché du lait si heureusement entrepris sous la poussée des événements, doivent être poursuivis; de leur réussite dépendent les solutions de deux problèmes d'ordre social et national particulièrement graves :

$1^{0}$ La réduction progressive de la mortalité infantile ;

$2^{\circ}$ L'amélioration de la situation économique de l'agriculture française. 
Dans ce travail, j'ai cherché à montrer comment sont dirigés les efforts, et pourquoi, en cette matière, tous les intérêts généraux en jeu sont convergents. Il m'apparaît donc que le problème posé peut être résolu, malgré les difficultés; qui, si elles existent réellement, ne sont pas insurmontables.

\section{PRÉSURES VÉGÉTALES. EXTRACTION ET PROPRIÉTÉS \\ par}

\section{CHRISTEN et E. VIRASORO}

(Institut de Recherches 'Scientifiques et Techniques de la Faculté de Chimie Industrielle. Université Nat. du Litoralle, Santa-Fé, R. Argentine. Directeur : Dr. H. Damianovich.)

$$
\text { (Fin) }
$$

Si l'on compare les courbes citées avec la figure $n^{0} 3$, qui traduit les résultats des expériences de Fleischmann [5] faites avec la présure animale, on voit dans l'une aussi bien que dans l'autre, que les temps diminuent progressivement jusqu'à atteindre un minimum

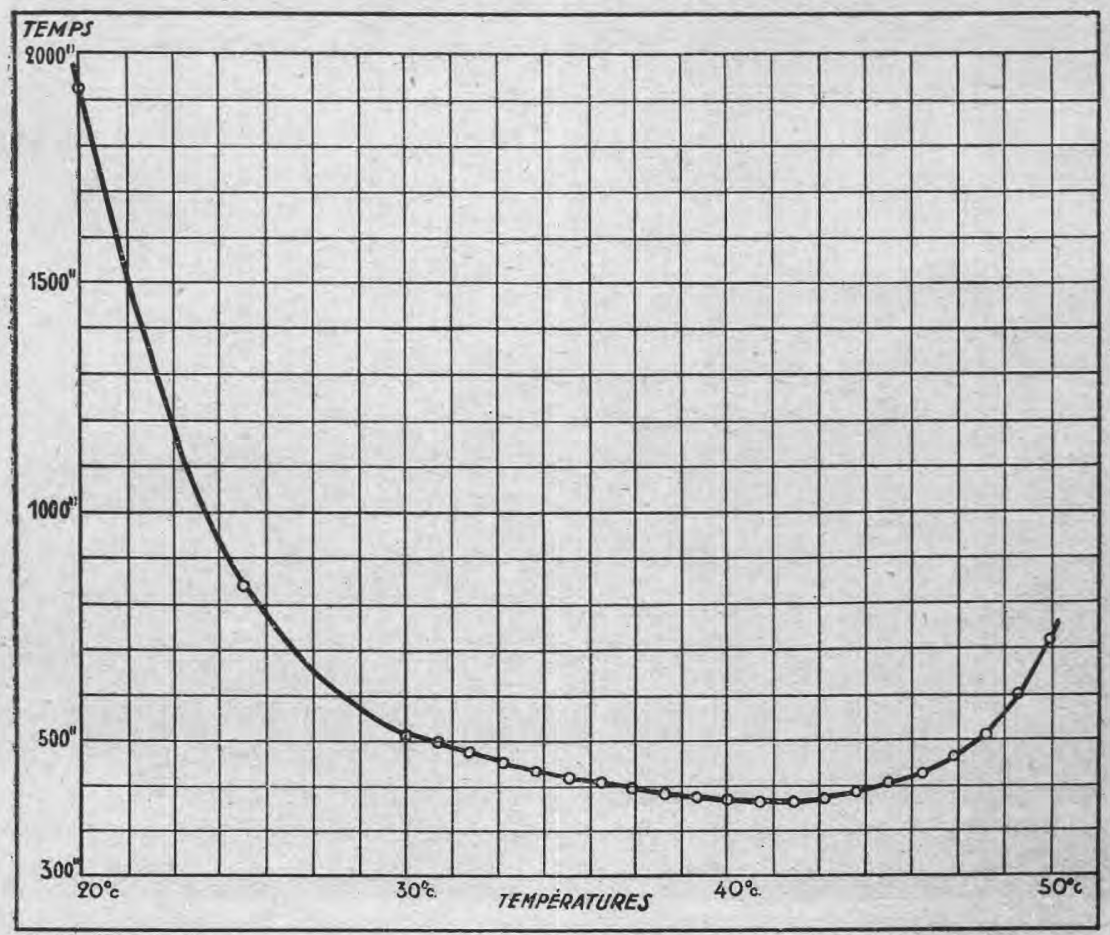

FigURE 3. 